\title{
Effect of Preparation Methods on the Particles Size, Dielectric Constant and Antibacterial Properties of ZnO Nanoparticles and Thin Film of ZnO/Chitosan
}

\author{
ASMA. M. AL-TURKI \\ Department of Chemistry, University of Tabuk, Faculty of Science, Tabuk, Saudi Arabia. \\ Corresponding author E-mail: aalturky@ut.edu.sa \\ http://dx.doi.org/10.13005/ojc/340163
}

(Received: Octuber 14, 2017; Accepted: December 23, 2017)

\begin{abstract}
In the present work, we have synthesized zinc oxide nanoparticles by two different methods simple solution route and sol- gel (Z1, Z2). SEM analysis of ZnO nanoparticles samples (Z1 and Z2) showed that, the morphology of samples found to be look like nanorods. Transmission Electron Microscopy (TEM) images showed that the particles are spherical, and the particles size of sample Z2 larger than sample Z1. The partial size of sample Z1 is between 17.61 to $2.81 \mathrm{~nm}$ and for sample Z2 was between 25 to $6 \mathrm{~nm}$. Also, thin films of $\mathrm{ZnO} /$ Chiosan nanocomposites (Z11and Z21) were prepared to study dielectric and antibacterial properties. Results showed that, the value of the dielectric constant for sample $Z 11$ is less than the value of $Z 21$, it is may be due to different in particles size according to the space-charge theory (dielectric constant increase with increasing of particles size). Frequency dependence dielectric properties of $\mathrm{ZnO} /$ Chiosan thin films (Z11 and Z21) at room temperature were measured. Dielectric constant $\varepsilon^{\prime}$ and dielectric loss $\varepsilon^{\prime \prime}$ for all samples were found to be decrease with increasing frequency.
\end{abstract}

The synthesized $\mathrm{ZnO} /$ Chiosan nanocomposite films exhibited activity against Gram-negative Pseudomonas aeruginosa (PA), and Gram-positive Staphylococcus aureus (SA).All results indicated that, the antimicrobial activity of the nanocomposite film samples due to presence of nano $\mathrm{ZnO}$ particles.

Keywords: Zinc oxide, Nanoparticles, Dielectric, Chitosan, Film.

\section{INTRODUCTION}

Nanocomposite has received increasing attention in recent years in industry. These materials have the ability to collect a group of properties in a single compound as mechanical, electric and optical properties. zinc oxide nanoparticles (NP) have the precedence of research consequent of their exclusive properties and wide applications ${ }^{1}$. Metal oxide nanoparticles synthesis by different methods such as: thermal decomposition, precipitation, hydrothermal, solvothermal, reverse micelles, wet

This is an $\mathbf{C}$ Open Access article licensed under a Creative Commons Attribution-NonCommercial-ShareAlike 4.0 International License (https://creativecommons.org/licenses/by-nc-sa/4.0/ ), which permits unrestricted NonCommercial use, distribution and reproduction in any medium, provided the original work is properly cited. 
chemical and sol gel method ${ }^{2,3}$. It has been verified anti-bacterial behavior of $\mathrm{ZnO}$ nanoparticles in many studies. The results showed that zinc oxide nanoparticles had an antimicrobial activity against both gram bacteria (Gram positive and Gram negative $)^{4}$. Zinc oxide (NP) also has an effect on microorganisms better than other metal oxides such as $\mathrm{SiO}_{2}$.

Polymers have many remarkable properties. With rapid technical development, polymers have replaced aluminum and iron for some purposes that require stress and high temperature. Over the past few years, there have been many researches on natural polymers such as Chitosan where it has been found to have biodegradability, low cost, and environmental suitability. Chitosan is commercially extracted from shrimp or crab shell ${ }^{5}$. Studies have shown that chitosan has an antibacterial activity that is resistant to many types of bacteria. Chitosan, an important polymer, has many chemical properties also, it used for immobilization of biomolecules due to good film forming ability, mechanical properties, non-toxicity, and low $\operatorname{cost}^{6}$. $\mathrm{ZnO}$ (NP) can be applied as aspect of organic/inorganic nanocomposite coatings in many industrial section, like food, packaging and medical care. ZnO (NP) have been inserted with chitosan, and polyimide to exhibit powerful antibacterial activity, dielectric behavior, and UV absorbance ${ }^{7}$.

In this study we studied the effect of synthesis methods of $\mathrm{ZnO}$ nanoparticles on the particles size, dielectric properties and antibacterial behavior. Simple solution route and sol- gel are the simple methods with good control of the structure and particle size at low temperature.

\section{EXPERIMENTAL}

\section{Material and Methods}

Chitosan with 85 percent degree of deacetylation, Ammonium hydroxide solution and absolute ethanol were purchased from Sigma Aldrich \& Co. Ltd (USA). Acetic acid, Glacial biochemical grade $99.86 \%$ was purchased from ACROS and Zinc chloride $97.6 \%$ was obtained from Holyland (Saudi Arabia). Two bacterial strains Pure cultures of Gram negative human bacteria viz.,
Pseudomonas aeruginosa and Gram positive bacteria viz., Staphylococcus aureus were obtained from "Culture Collection of Antibiotic Resistant Microbes (CCARM)" Military Hospital Tabuk. All the chemicals were analytical grade and used without further purification.

\section{Synthesis of ZnO (NP) powder First Method (simple solution route)}

A round bottom was used to dissolved $1.35 \mathrm{~g}$ of $\mathrm{ZnCl}_{2}$ in mixer of water $(80 \mathrm{~mL})$ and ethanol $(20 \mathrm{~mL})$ then added $5 \mathrm{~mL}$ of $\mathrm{NH}_{4} \mathrm{OH}$ drop by drop for $60 \mathrm{~min}$. at $60^{\circ} \mathrm{C}$ with stirring for three hours. The powder was dried in the oven at $200^{\circ} \mathrm{C}$ for $24 \mathrm{~h}$ to obtain the nanoparticle powder of zinc oxide (Z1).

\section{Second Method(sol-gel)}

A round bottom was used to dissolved $1.35 \mathrm{~g}$ of $\mathrm{ZnCl}_{2}$ in mixer of water $(20 \mathrm{~mL})$ and $\mathrm{NH}_{4} \mathrm{OH}$ $(5 \mathrm{~mL})$ then added $80 \mathrm{~mL}$ of ethanol drop by drop for $60 \mathrm{~min}$. at $60^{\circ} \mathrm{C}$ with stirring for three hours. The powder was dried in the oven at $200{ }^{\circ} \mathrm{C}$ for $24 \mathrm{~h}$ to obtain the nanoparticle powder of zinc oxide (Z2).

\section{Preparation of ZnO/Chitosan nanocomposite Films}

Chitosan solution was prepared by dissolving $1 \mathrm{~g}$ of chitosan in $100 \mathrm{ml}$ of $2 \%(\mathrm{v} / \mathrm{v})$ aqueous glacial acetic acid with the assistance of a magnetic stirrer at $100{ }^{\circ} \mathrm{C}$ for 4 hours. A $\mathrm{ZnO} /$ Chitosan nanocomposite film was prepared by mixed $10 \mathrm{~mL}$ of Chitosan solution with $0.05 \mathrm{gm}$ of $\mathrm{ZnO}$ nanoparticles and stirring for $3 \mathrm{~h}$ a room temperature. Then it was casted at room temperature in petri dish until it dried and formed film (Z11 and Z21).

\section{Characterization and measurements Detection Method}

From Scanning Electron Microscope (SEM) and Energy Dispersive $\mathrm{X}$-ray Analysis (EDAX) the samples were prepared by attaching the particles to $12.5 \mathrm{~mm}$ diameter AI SEM stubs having sticky $12 \mathrm{~mm}$ diameter $\mathrm{C}$ tabs. The samples were viewed by FEI Quanta 200 SEM (Instrument ID: AN_SEM-2) operated at settings: Accelerating Voltage $=20.0 \mathrm{kV}$, Working Distance $=10 \mathrm{~mm}$, Spot Size=4.5 (EDX), 2.0-3.0 (SEM). The images were digitally recorded in secondary electron imaging mode at different magnifications at resolution setting of $1024 x 884$ pixels. EDX analysis was performed 
on multiple particles in area mode $\left(\sim 25-50 \mathrm{~m}^{2}\right)$ using EDAX Genesis XM4 system attached to SEM. The elemental contents were determined using standardless ZAF option.

The Transmission Electron Microscopy (TEM) studies were performed (TEM, JEOL JEM$3010,300 \mathrm{kv}$ ). The samples for TEM were prepared by making suspension from the powder in deionized water. A drop of the suspension was put into the carbon gride and left to dry.

The electrical properties was measured at room temperature by (Hioki, LCR Hitester 353250). The frequency dependence of electrical properties for prepared samples were measured from $50 \mathrm{~Hz}$ to $5 \mathrm{Mhz}$.

In vitro antimicrobial activity was examined for nanocomposite films of $\mathrm{ZnO} /$ Chiosan (Z11, Z21). Pure cultures of Gram negative human bacteria viz., Pseudomonas aeruginosa and Gram positive bacteria viz., Staphylococcus aureus were obtained from "Culture Collection of Antibiotic Resistant Microbes (CCARM)" Military Hospital Tabuk. The bacterial strains were maintained and grown in a nutrient agar medium.

\section{RESULTS AND DISCUSSION}

\section{EDAX, SEM and TEM study}

EDAX analysis was performed on multiple
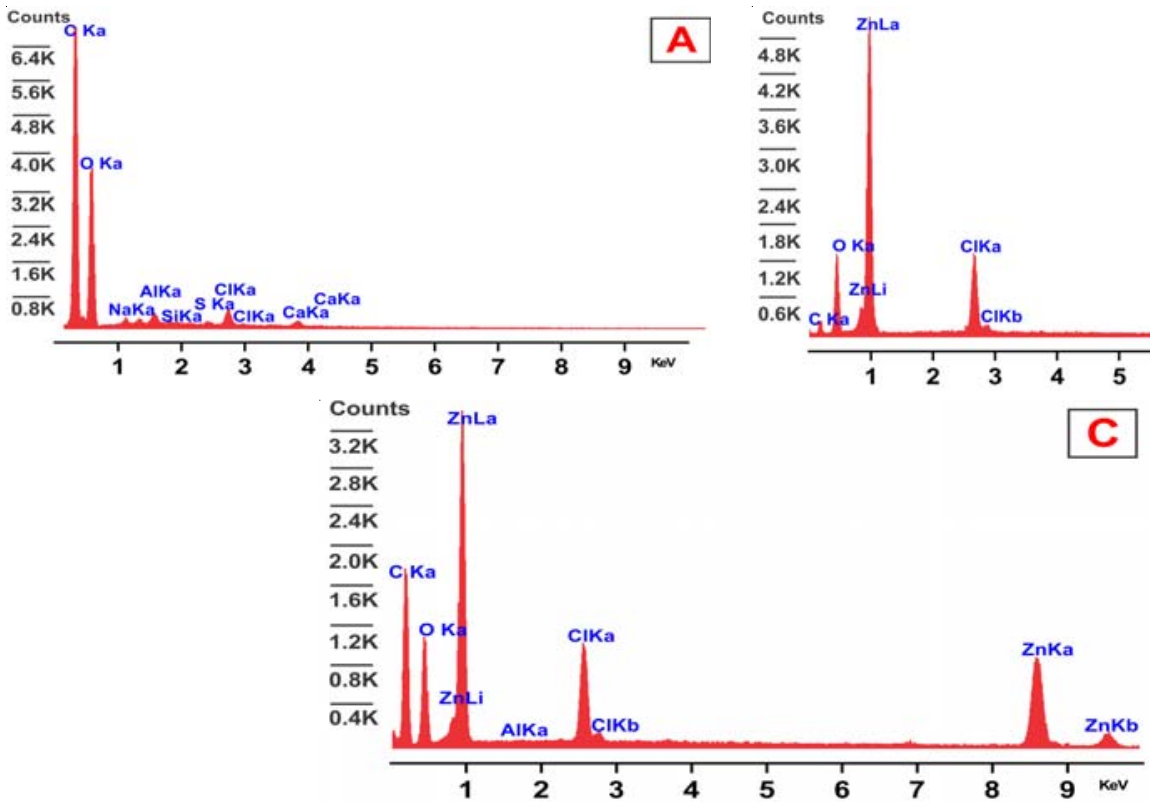

Fig.1. The EDAX images for (A) pure Chitosan, (B) for Z1 and (C) for Z2 particles in area mode $\left(\sim 25-50 \mathrm{~m}^{2}\right)$ using EDAX Genesis XM4 system attached to SEM. The elemental contents for pure Chitosan film and (Z11, Z21)were determined using standardless ZAF option shows in Fig.1(a, b, c). SEM analysis Morphology and structure of $\mathrm{ZnO}$ nanoparticles samples (Z1 and Z2) were further investigated by SEM analysis. The SEM micrographs of the $Z 1$ and $\mathrm{Z} 2$ nanoparticles are shown in the Fig. (2). It shows that the synthesized $\mathrm{ZnO}$ nanoparticles are well dispersed. Fig.(2a,b) shows the SEM images for the prepared sample(Z1), the morphology of samples found to be look like nano rods. Also Fig. (2c,d) shows the SEM images for the prepared sample(Z2), the morphology of samples also found to be as rods. TEM images are used to study the microstructures of the prepared samples. The SEM results were well supported by TEM measurements. Fig ( 3 a, b, c and d) shows the representative TEM images of the prepared samples. The morphology of the all samples were found to be nearly spherical but it look like a rod due to agglomeration of nanoparticles, The result of TEM shows that, the partials size of sample Z2 larger than sample Z1. The partials size of sample Z1 was between 17.61 to $2.81 \mathrm{~nm}$ and for sample $Z 2$ was between 25 to 6 $\mathrm{nm}$. TEM results indicated dark sites may be due to the accumulation of nanoparticles ${ }^{8}$.

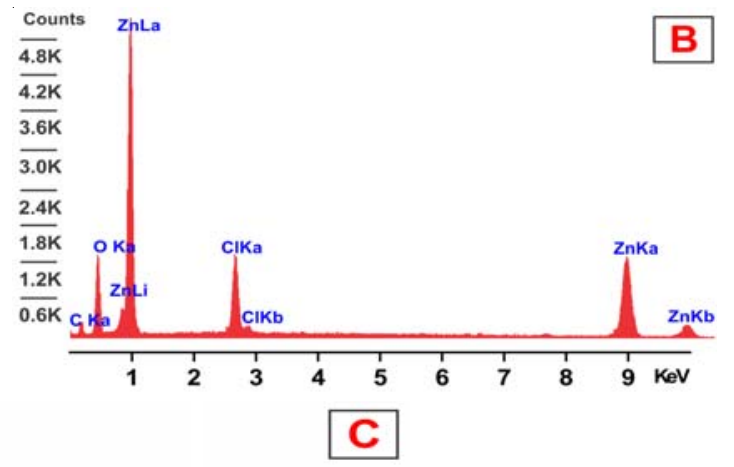



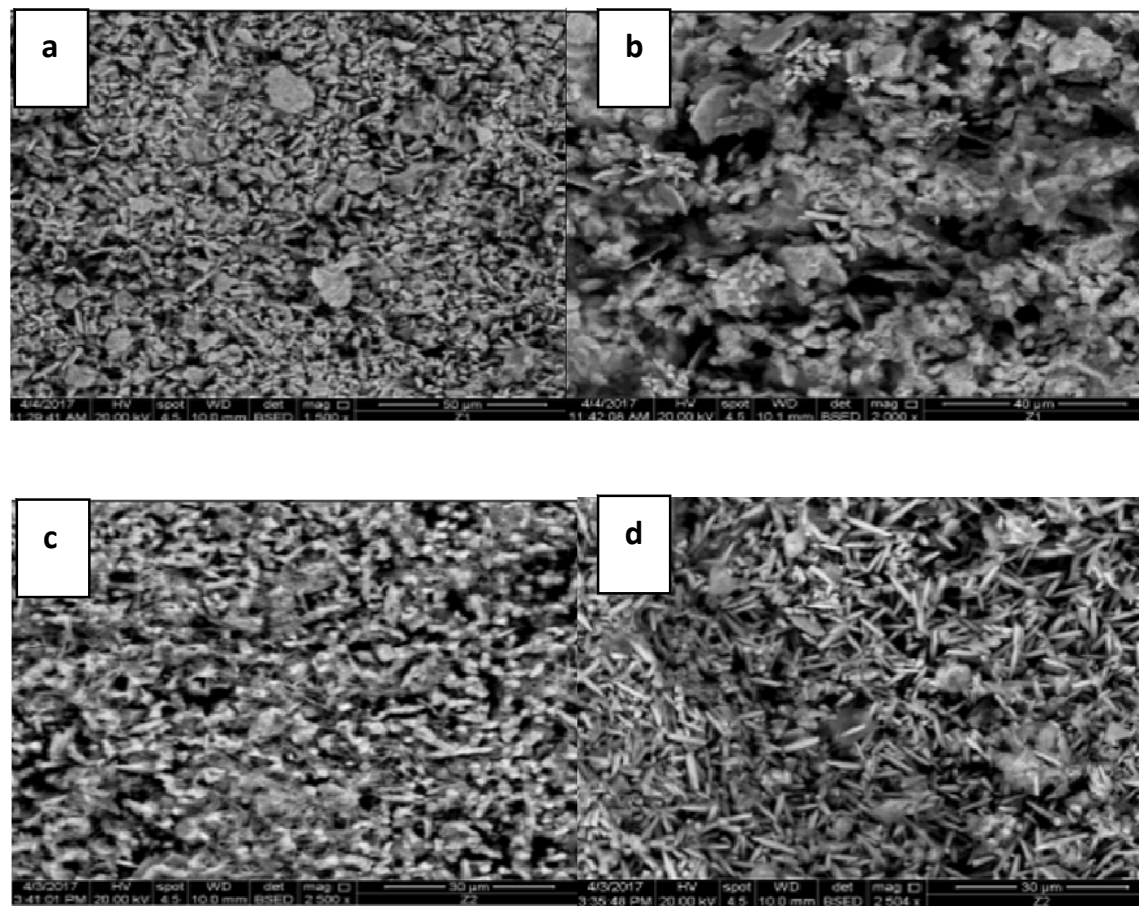

Fig. 2. (1a,b) The SEM images for sample(Z1) and .(2c,d) The SEM images for sample(Z2)
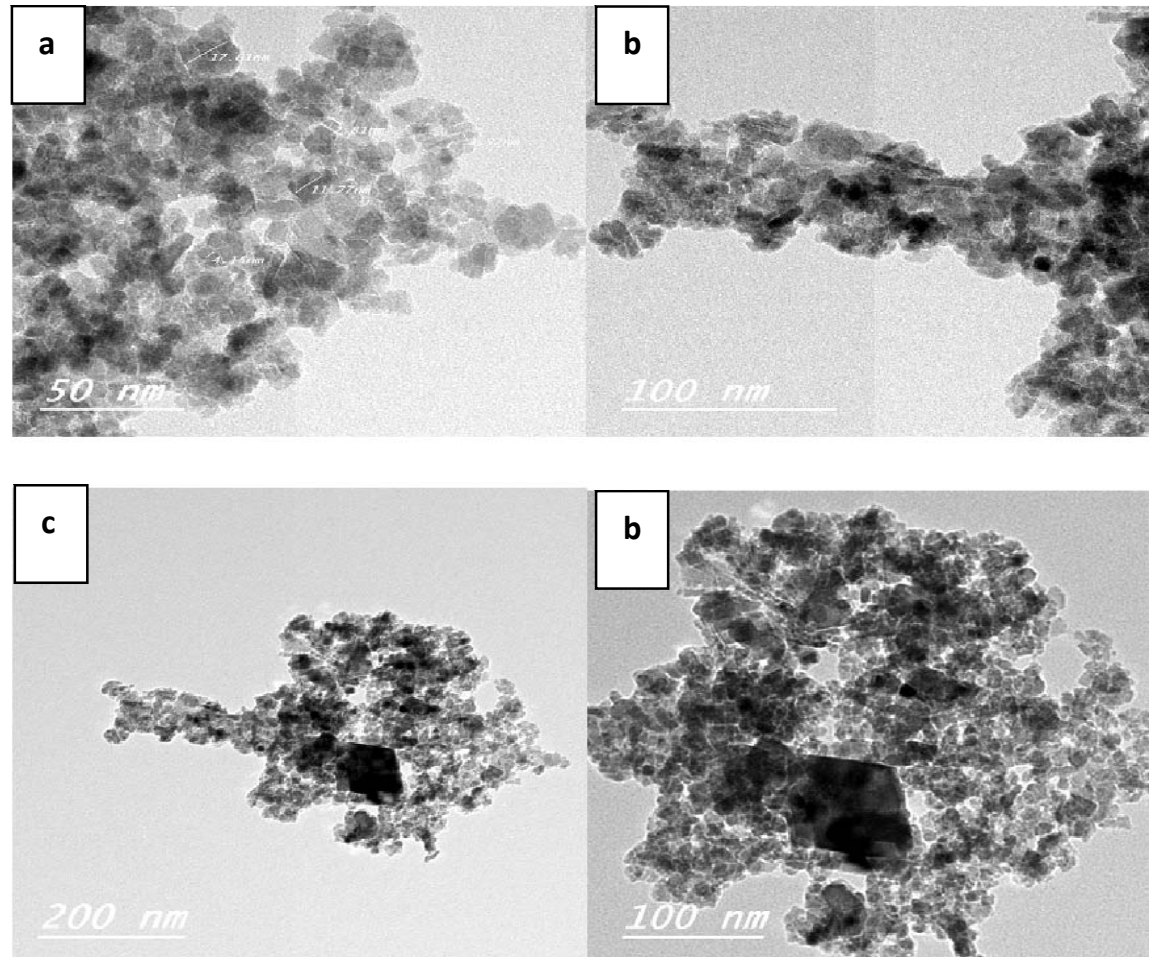

Fig. 3. (3a, b) The TEM images for sample(Z1) and .(3c,d) The TEM images for sample(Z2) 


\section{Dielectric Properties}

We studded dielectric constant $\left(\varepsilon^{\prime}\right)$ and dielectric loss ( $\left.\varepsilon^{\prime \prime}\right)$ frequency of pure Chitosan and nanocomposite films of Chitosan/ZnO were prepared by two different methods $(Z 11, Z 21)$, all samples collected at room temperature in the frequency range $(50 \mathrm{~Hz}$ to $5 \mathrm{MHz})$ as shown in Fig.4. Show the relation between the frequency and Dielectric constant $(\varepsilon$ ') for all samples (pure Chitosan, Z11 and Z21). The spectrum for all samples show that dielectric constant ( $\left.\varepsilon^{\prime}\right)$ decreased with frequency value increased. Variation of dielectric constant with frequency in Fig.4a at room temperature (298K) for all samples, we found that the dielectric constant decreased with increased of frequency. Also, at high frequencies the dielectric constant $\left(\varepsilon^{\prime}\right)$ oncoming a limiting constant value. At low frequency the dielectric constant is dependent on polarization effects (electronic-ionic-orientation polarization), but at high frequencies $\left(\varepsilon^{\prime}\right)$ is dependent on the electronic polarization only, so that the value of dielectric constant are less ${ }^{10}$.

In Fig. 4a it obviously seen that, dielectric constant of pure chitosan film is higher than that of all nanocomposite films of chitosan/ZnO samples $(Z 11, Z 21)$, The possibility that $Z n$ ions in all samples slow down the comparatively fast segmental motion of Chitosan due to "nano effect"11. So, if nano particles were added, the dielectric constant of a polymer composite would decrease. Also, the value of the dielectric constant for sample Z11 was less than the value of $Z 21$, it is may be due to different in particles size (dielectric constant increase with increasing of particles size).We can explain that according to the space-charge theory from Okazaki and Nagata ${ }^{12}$, The space charge sites inside particles boundaries and domain wall is cause forming electric field which effects the motion of domain walls. The surface area of the spacecharge decrease when the particles size increases. So, the space-charge field will decrease. The domain walls are completely free in large particles, as well as dielectric constant increases for samples with large particles size. Fig. 4b showing dielectric loss å" as a function of frequency for pure chitosan and nano composite films of Chitosan/ZnO were prepared by two different methods $(Z 11, Z 21)$ at room temperatures shows a relaxation peaks for $(Z 11, Z 21)$. The appearance of relaxation time is due to the efforts done by ionic charge transport in the $\mathrm{ZnO}$ nanoparticles in $\mathrm{Z11}$ and $\mathrm{Z} 21$ to follow the change in the direction of applied field ${ }^{10}$. We calculated the relaxation time from the following equation:

$$
\tau=1 / 2 \pi \int_{\mathrm{m}}
$$

where $\tau$ is the relaxation time and $\int_{\mathrm{m}}$ is the frequency of the maximum dielectric loss $\varepsilon$ ". The relaxation parameters for samples $Z 11$ and Z21 are in Table. 1.

Table. 1: relaxation time value

\begin{tabular}{lll}
\hline Sample & $\tau$ (second) & $\log \tau$ \\
\hline Z11 & 0.000796 & -3.0989 \\
Z21 & 0.000637 & -3.1959 \\
\hline
\end{tabular}

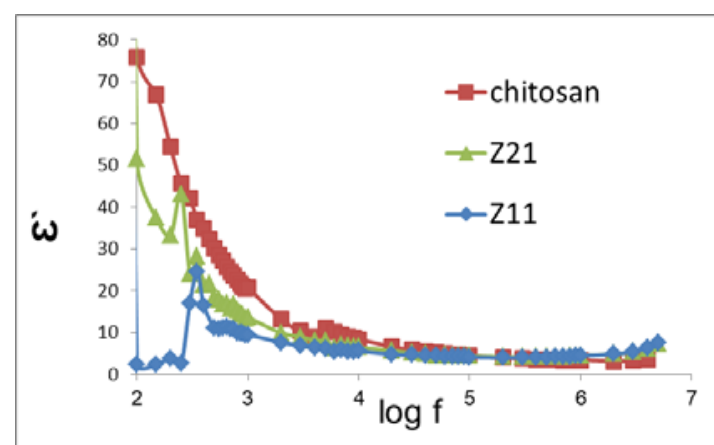

(a)

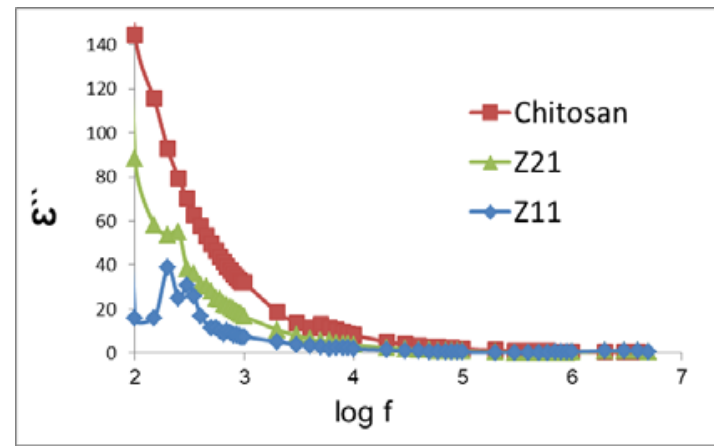

(b)

Fig.4. (4a) dielectric constant as a function of frequency and (3b) dielectric loss å" as a function of frequency for pure chitosan, Z11 and Z21 at room temperatures 


\section{Antimicrobial behavior}

The antimicrobial activities of nanocomposite films of Chitosan/ZnO (Z11, Z21) have tested against two different types of microorganisms by using disc diffusion method. For the determination of antimicrobial activity of nanocomposite films of Chitosan/ ZnO (Z11, Z21), the Gram-negative Pseudomonas aeruginosa(PA), and Gram-positive Staphylococcus(SA) aureus(PA) were used. Mueller Hinton Agar (MHA) media was prepared and poured into sterilized Petri plates and then plates were spreader with of the bacteria separately. The loaded disks were applied carefully to the surface of the agar plates using sterile forceps. The experiment was carried out and the diameters of the zones of inhibition were measured after $24 \mathrm{~h}$ of incubation at $37{ }^{\circ} \mathrm{C}$. Standard antimicrobial agents including Gentamicin (100 mg/mL) was used as control ${ }^{13}$.
In this study, the antibacterial activity of nanocomposite films of Chitosan/ZnO (Z11, Z21) against Gram-negative Pseudomonas aeruginosa, and Gram-positive Staphylococcus aureus was studied qualitatively by halo method (zone of inhibition). As shown in Fig. 5, Z11 and Z21 show antimicrobial activity, Where we note that a clear zone of inhibition within and around the nanocomposite films in both samples, but the area of the inhibition effect of nanocomposite films for the films in the case of sample Z11and Z21 with Gram-positive Staphylococcus aureus larger than the sample Z11 and Z21 with Gram-negative $P$ seudomonas aeruginosa. All results indication that the antimicrobial activity of the nanocomposite film samples due to presence of $\mathrm{ZnO}$ nano particles. It is believed that $\mathrm{ZnO}$ nano particles release reactive oxygen species that work with $\mathrm{Zn}$ ions to aggression the negatively charged cell wall and will drive to death of bacteria ${ }^{14}$.
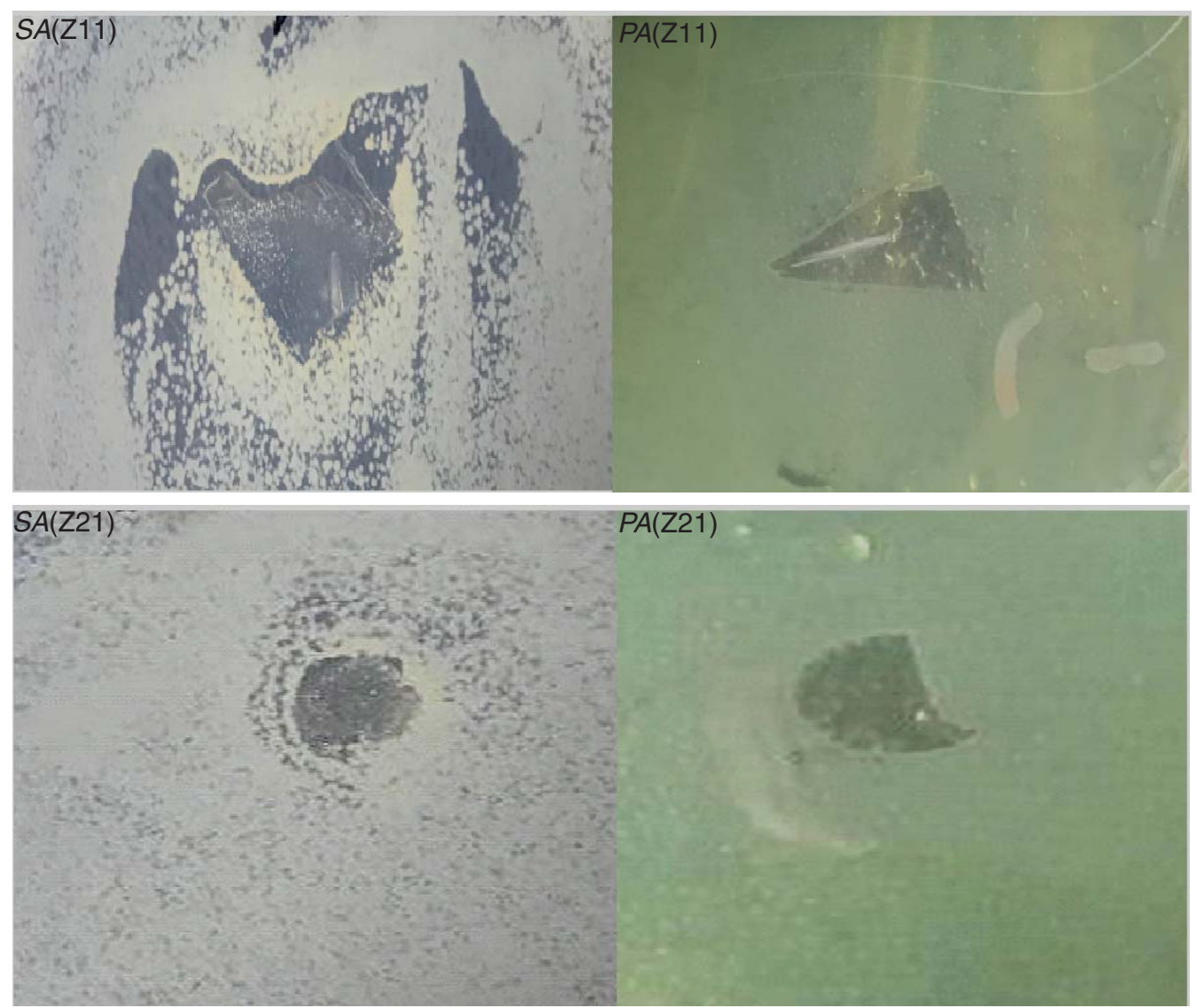

Fig.5. The antimicrobial activity of nanocomposite film of $(\mathrm{Z11}, \mathrm{Z21})$ against Gram-negative Pseudomonas aeruginosa (PA), and Gram-positive Staphylococcus aureus (SA) 


\section{CONCLUSION}

$\mathrm{ZnO}$ nanoparticles with spherical structure have been synthesized successfully by two different methods.

The partials size of sample Z2 larger than sample Z1. The partials size of sample Z1 was between 17.61 to $2.81 \mathrm{~nm}$ and for sample Z2 was between 25 to $6 \mathrm{~nm}$.

The dielectric measurements indicate that the dielectric constant of pure Chitosan and $\mathrm{ZnO}$ nanoparticles $(Z 1, Z 2)$ decrease, in the highfrequency range.

The value of the dielectric constant for sample Z11 is less than the value of Z21, it is may be due to different in particles size according to the space-charge theory (dielectric constant increase with increasing of particles size).
The synthesized Chitosan/ZnO nano composite films exhibited activity against Gram-negative, Pseudomonas aeruginosa (PA), and Gram-positive Staphylococcus aureus (SA).

\section{ACKNOWLEDGEMENT}

The author would like to acknowledge financial support for this work, from the Deanship of Scientific Research (DSR), University of Tabuk, Tabuk, Saudi Arabia, under grant no. S-1438-0251. The author is deeply indebted to professor. Magdah Dawy and Dr. Rabab Khaled at National Research Center, Cairo, Egypt, for their help in Dielectric Analysis, Also, Mr. Abdullah Almayouf from Analytical Lab at SABIC Technology \& Innovation Center for his inspiring help in Surface Morphology and Elemental Analysis for samples. Author acknowledges Dr. Jayda G. Eldiasty, in Biology Department at university of Tabuk for her assistance in Antimicrobial test.

\section{REFERENCES}

1. Rouhi, J.; Mahmud, S.; Naderi, N.; Raymond, C.; Mahmood, M. Nano. Res. Lett. 2013, 8 , 364-370.

2. Rabenau, A., Ang. Chem. Inter. Edition in English 1985, 24, 1026-1040.

3. Huang, J.; Matsunaga, N.; Shimanoe, K.; Yamazoe, N.; Kunitake, T., Chem. of Mat. 2005, 17, 3513-3518.

4. Xie, Y.; He, Y.; Irwin, P. L.; Jin, T.; Shi X., appl. And env. Micro. 2011, 77, 2325-2331.

5. Su, C. H.; Sun, C. S. ; Juan, S. W. ; Hu, C. H.; Ke, W. T. ; Sheu M. T., Biomaterials, 1997, 18:1169-1174.

6. Farouk, A.; Moussa, S.; Ulbricht, M.; Textor, T., Inter. J. of Carb. Chem. 2012, 8.

7. Ma, J. ; Zhu, W.; Tian, Y.; Wang, Z., Nano. Res. Lett. 2016, 11, 200-208.
8. Udayakumar, S.; Renuka, V.; Kavitha, K., J.Chem. Pharm. Res. 2012, 4, 1271-1280.

9. Abd El-razek, A. A.; Saed1, E. M.; Gergs, M. K., IOSR J. of Appl. Phys. 2014, 6, 20-29.

10. ELmezayyen, A. S.; Reicha, F.M., Open J. of App. Sci. 2015, 5, 415- 427.

11. Yang, W.;Yi, R.;Yang, X.; Xu, M.; Hui, S.; Cao, $\mathrm{X}$., Trans. On electrical and electronic mat. 2012, 13, 116-120.

12. Okazaki, K.; Nagata, K. J. Am. Ceram. Soc.,1973, 56, 82-86.

13. Lalitha, V.; Kiran, B.; Raveesha, K. A., Pharm. 2011, 2, 78-85.

14. Gordon, T.; Perlstein, B.; Houbara, O.; Felner, I.; Banin, E.; Margel, S., Colloids and Surfaces A: Phys. Eng. Asp. 2011, 374, 1-8. 